MAX-PLANCK-INSTITUT FÜR GESELLSCHAFTSFORSCHUNG MAX PLANCK INSTITUTE FOR THE STUDY OF SOCIETIES

MPIfG Working Paper 97/9, November 1997

\title{
Demokratische Politik in der internationalisierten Ökonomie[1]
}

\author{
von Fritz W. Scharpf $\equiv$
}

Fritz W. Scharpf ist Direktor am Max-Planck-Institut für Gesellschaftsforschung, Köln 1 Entgrenzte Ökonomie, beschränkte Politik

Demokratische Politik und kapitalistische Ökonomie koexistieren in einer antagonistischen Symbiose, die erst nach der großen Weltwirtschaftskrise in den Jahrzehnten nach dem Zweiten Weltkrieg verträgliche Konfigurationen entwickelt hat. Die Politik hatte gelernt, die kapitalistischen Krisen zu dämpfen und die Ausbeutung der menschlichen Arbeitskraft und der Natur zu begrenzen, ohne die innovative Dynamik der Marktwirtschaft zu lähmen. Zugleich erlaubten die Erträge des kapitalistischen Wachstums den Ausbau sozialstaatlicher Sicherungen und marktkorrigierender Leistungen, die ihrerseits die bis dahin ja durchaus prekäre Legitimation der demokratischen Regierungsform so verstärkten, daß am Ende des Jahrhunderts konkurrierende Legitimitäts-Prätentionen nirgendwo mehr vertreten werden.

Notwendige Voraussetzung dieser "demokratischen Zivilisierung der kapitalistischen Ökonomie" war die in den Nachkriegsjahrzehnten zunächst gewährleistete Kontrolle des Nationalstaats über seine ökonomischen Außengrenzen. Sie erlaubte es der staatlichen Zinspolitik, die Mindestrendite von Finanzanlagen zu variieren, und so die relative Attraktivität von arbeitsplatzschaffenden Realinvestitionen im Sinne einer keynesianischen Vollbeschäftigungspolitik zu beeinflussen. Innerhalb kontrollierter Außengrenzen war der staatliche Gesetzgeber (ebenso wie die Tarifpolitik der Gewerkschaften) überdies in der Lage, einheitliche arbeitsrechtliche, sozialrechtliche oder umweltrechtliche Regeln für alle miteinander in unmittelbarer Konkurrenz stehenden Unternehmen vorzuschreiben - und weil alle Konkurrenten in gleichem Maße betroffen waren, konnte jeder von ihnen die Kosten solcher Regelungen auf die Preise überwälzen, so daß die Rendite des eingesetzten Kapitals dadurch nicht gefährdet wurde. Soweit unter diesen Bedingungen die internationale Wettbewerbsfähigkeit der einheimischen Wirtschaft überhaupt zum Problem wurde (etwa bei chronischen Defiziten der Zahlungsbilanz), bot schließlich die Wechselkurspolitik ausreichende Korrekturmöglichkeiten.

Seit Mitte der siebziger Jahre haben die Nationalstaaten jedoch die Kontrolle über ihre ökonomischen Außengrenzen wieder aufgegeben oder verloren. Die Finanzmärkte haben sich globalisiert, und das tägliche Volumen der grenzüberschreitenden Finanztransfers übersteigt heute die Summe der Devisenreserven aller Nationalbanken. Der Handel wurde so weit liberalisiert, daß heute die Konsumenten unter Gütern und Dienstleistungen, die irgendwo auf der Welt produziert wurden, fast ohne Einschränkung frei wählen können. Zumindest innerhalb der Europäischen Union haben deshalb auch die Unternehmen die völlig freie Wahl unter den Produktionsstandorten in allen Mitgliedstaaten, ohne daß dadurch der Zugang zum heimischen Markt in irgendeiner Weise gefährdet würde. Überdies wird in der Europäischen Union die Liberalisierung nun auch in Bereichen 
vorangetrieben, die - wie die Telekommunikation, der Luftverkehr, der

Straßengüterverkehr oder die Energieversorgung - bisher auch im nationalen Rahmen dem Marktwettbewerb entzogen waren, und zugleich sind die Mitgliedstaaten strikten Regeln der "negativen Integration" unterworfen, die wettbewerbsverzerrende Maßnahmen jeglicher Art ausschließen sollen (Scharpf 1996).

Mit der Internationalisierung der Kapitalmärkte hat die nationale Politik kaum noch die Chance, Massenarbeitslosigkeit durch Globalsteuerung zu verhindern. Zugleich verminderte sich mit der Liberalisierung des Handels und der freien Standortwahl der Unternehmen die Fähigkeit des Staates und der Gewerkschaften, die Ausbeutung von Arbeitskraft und Natur durch marktbeschränkende Regulierung zu begrenzen,[2] und die kapitalistische Verteilung von Lebenschancen mit den Mitteln der Steuer- und Sozialpolitik oder der Lohnpolitik zu korrigieren. Der Staat mag zwar noch über das Monopol legitimer Gewaltsamkeit verfügen, aber er verfügt nicht mehr über das Regelungsmonopol. Statt dessen können nun die Wirtschaftssubjekte die neuen Freiheiten des grenzüberschreitenden Verkehrs von Waren, Dienstleistungen, Kapital und Arbeitskräften nutzen, um den aus einzelwirtschaftlicher Sicht jeweils günstigsten Standort zu wählen, während die Staaten untereinander zum "Standortwettbewerb" und damit zum Wettbewerb der Regulierungssysteme um mobile Produktionsfaktoren und Steuerzahler gezwungen werden.

Soweit die heute herrschende Diagnose, gegen die freilich der Vorwurf der Übergeneralisierung erhoben werden kann. Selbst auf den Kapitalmärkten bleibt der weit überwiegende Teil aller Anlagen nach wie vor in den nationalen Grenzen. Aber die erwarteten und am Markt durchsetzbaren Renditen orientieren sich nun dennoch am internationalen Niveau. Ebenso ist es richtig, daß Export- und Importquoten sich derzeit nicht mehr stark erhöhen, aber auch das ändert nichts daran, daß die gesamte Industrieproduktion und große Teile der Dienstleistungen nun unter intensiverem Wettbewerbsdruck stehen, und daß der Spielraum für die Überwälzung von Steuern, Regulierungskosten und Arbeitskosten geringer geworden ist, als dies noch vor zwei Jahrzehnten der Fall war. Schließlich trifft es auch zu, daß das jetzt erreichte Niveau der ökonomischen Globalisierung keineswegs ein historisch neues Phänomen darstellt. In der Tat wurde die vor dem Ersten Weltkrieg und in den zwanziger Jahren erreichte Integration der Kapitalmärkte erst im letzten Jahrzehnt wieder überschritten, und das gleiche gilt für die industriellen Direktinvestitionen im Ausland und für den Außenhandel (Hirst/ Thompson 1996).

Aber auch darin wird man kaum einen Trost sehen können. Der internationalisierte Kapitalismus des letzten Jahrhunderts produzierte rasches Wachstum und tiefe Krisen, riesige Vermögen und äußerste Verelendung - eben all die Folgen, die Marx und Engels beschrieben hatten. Sie mußten von den Betroffenen ertragen werden, weil die staatliche Politik entweder gar nicht oder nur in sehr beschränktem Maße dem demokratischen Einfluß der Betroffenen ausgesetzt war. Nach dem ersten Weltkrieg hatte sich das geändert, und so manifestierte sich die ökonomische Weltwirtschaftskrise der frühen dreißiger Jahre überall auch als politische Krise und in Deutschland als Katastrophe der demokratischen Legitimität. Die produktive Symbiose von politischer Demokratie und kapitalistischer Ökonomie jedenfalls wurde erst in den Nachkriegsjahrzehnten und unter dem Schutz kontrollierbarer Außengrenzen erreicht, und sie wird erst jetzt auf eine ernsthafte Bewährungsprobe gestellt.

In der neoliberalen Literatur zur politischen Ökonomie der Globalisierung wird der Abbau der Außengrenzen begrüßt, weil der Zwang zum Wettbewerb der politischen Systeme die hypertroph gewordene Staatsmacht beschränkt und die Freiheit der Märkte gegen politische Interventionen schützt (Streit/ Mussler 1994; Streit 1996). Andere Autoren kommen zu dem Schluß, daß mit dem unvermeidlichen Verlust nationalstaatlicher Steuerungspotentiale gegenüber der globalisierten Ökonomie 
(Cerny 1990; 1993; 1993; Streeck 1997) auch das "Ende der Demokratie" (Guéhenno 1994) abzusehen sei; während wieder andere darauf setzen, daß die Verluste der nationalen Politik durch den Aufbau supranationaler Regelungskompetenzen auszugleichen seien (Däubler 1989; Delors 1993; Leibfried/ Pierson 1995; Bercusson 1996; Sassoon 1996; Larsson 1997), die ihrerseits durch Strukturen einer europäischen oder "kosmopolitischen Demokratie" (Held 1993; 1995) legitimiert werden können.

Mir scheint die erste Antwort normativ defizitär. Sie unterschätzt, wie ich an anderer Stelle zu zeigen versucht habe, die wohlfahrtstheoretische Bedeutung marktbeschränkender und marktkorrigierender Staatsfunktionen (Scharpf 1997a). Darauf will ich hier nicht näher eingehen. Meine Einwände gegen die zweite und die dritte Antwort sind empirischer Art: Die zweite erscheint mir zu pessimistisch, weil sie den Spielraum für nationale Lösungen unterschätzt, die auch gegenüber dem verschärften internationalen Wettbewerb robust sind. Die dritte Antwort erscheint mir zu optimistisch, weil sie nicht nur die faktischen, sondern auch die legitimatorischen Schranken der europäischen - und erst recht der internationalen - Politik unterschätzt. Eine normativ begründbare und empirisch zutreffende Einschätzung des Problemlösungspotentials und der Legitimationsprobleme der europäischen (und globalen) Mehrebenenpolitik wird freilich erschwert durch unübersichtliche Diskurse über das "europäische Demokratiedefizit". An der einen Front wird darüber gestritten, ob oberhalb der Nationalstaaten ein "europäischer Demos" vorausgesetzt werden dürfe, auf den erst demokratische Legitimation sich beziehen könnte (Grimm 1995; Habermas 1995; 1996; Weiler 1995; 1996; Kielmansegg 1996; Böckenförde 1997). Fast völlig unabhängig von dieser Kontroverse haben sich optimistischere Diskurse entwickelt, in denen sich die Hoffnung auf formal institutionalisierte Formen einer "kosmopolitischen Demokratie" offenbar umstandslos verbinden mit Projekten der zivilgesellschaftlichen Selbstorganisation (Cohen/ Rogers 1992; Dettling 1997; Dubiel 1997) und mit den Idealen und Realitäten von deliberativer Politik (Habermas 1996; Daele/ Neidhardt 1996) und von Problemlösungen in Verhandlungsnetzwerken (Pitschas 1994; Schmalz-Bruns 1995; Benz/ Lehmbruch 1996; Heinelt 1996; Joerges/ Neyer 1997). Die Unterschiedlichkeit dieser Diskurse legt es nahe, zunächst das Thema einzugrenzen und dann vor der Erörterung spezifischer Legitimationsprobleme der europäischen Politik eine normativanalytische Klärung der Grundlagen demokratischer Legitimation zu versuchen.

\section{Demokratie als Verfahren zur Legitimation von Herrschaft}

An den Anfang stelle ich zwei Ausgrenzungen. Zum einen ist der hier verwendete Demokratiebegriff instrumentell, nicht expressiv gemeint. Im folgenden geht es also nicht um demokratische Partizipation als Selbstzweck (Lindner 1990) oder als Weg zur vollen Entfaltung der allseits gebildeten Persönlichkeit, sondern um die Ermöglichung und Kontrolle kollektiven Handelns. Zum zweiten unterscheide ich zwischen Verwendungen des Demokratiebegriffs im Sinne eines

Organisationsprinzips zivilgesellschaftlicher Interaktionen und seiner Verwendung zur Legitimation staatlicher Herrschaft. In der Sache ist selbstverständlich gegen die kommunitaristische Wiederentdeckung eines zwischen Markt und Staat liegenden Bereichs des freiwillig-gemeinsamen Handelns ebensowenig einzuwenden wie gegen die Vermutung, daß in der Zivilgesellschaft noch ungenutzte

Problemlösungspotentiale mobilisiert werden könnten, welche in bestimmten Bereichen die abnehmende Handlungsfähigkeit des Staates kompensieren könnten.[3] Diese Perspektive kritisiere ich nicht, aber sie bleibt hier ausgeblendet. Statt dessen beginnen meine weiteren Überlegungen mit der Prämisse der altmodischen Wohlfahrtstheorie, derzufolge es eine Klasse von wohlfahrtsrelevanten 
Leistungen (oder Problemlösungen) gibt, die weder durch das einseitige Handeln autonomer Individuen, noch durch den Markt - und eben auch nicht durch freiwilliggemeinsames Handeln in der Zivilgesellschaft - produziert werden können. Dazu gehören insbesondere auch die zuvor erwähnten marktbeschränkenden und marktkorrigierenden Regelungen, die erst die kapitalistische Ökonomie sozialverträglich haben werden lassen. Wenn derartige Regelungen überhaupt wirksam werden sollen, dann sind sie direkt oder indirekt angewiesen auf die - vom Monopol legitimer Gewaltsamkeit gestützte - Rechtsetzungs- und Steuererhebungskompetenzen des Staates.

Aber der Einsatz herrschaftlicher Kompetenzen bleibt ineffektiv und jedenfalls ineffizient, wenn er nicht vom "Legitimitätsglauben" der Adressaten (und insbesondere der diese beobachtenden und mit diesen kommunizierenden Bezugsgruppen) gestützt wird. Dieser erst sichert, in Max Webers Formulierung, die Chance des Gehorsams in der Weise, "daß das Handeln des Gehorchenden im wesentlichen so abläuft, als ob er den Inhalt des Befehls um dessen selbst willen zur Maxime seines Verhaltens gemacht habe" (Weber 1956, 123). Wo dieser Legitimationsglaube fehlt, bleibt die Folgebereitschaft auf erzwingbare und kontrollierbare Verhaltensweisen beschränkt - mit dem Ergebnis, daß der Sanktionsaufwand steigt, während dennoch Regelverstöße, Korruption, Steuerhinterziehung und Kriminalität um sich greifen. Legitimität hat also höchst reale Vorteile und ihre Erosion wird teuer bezahlt. Was aber kann den Legitimitätsglauben begründen?

Max Weber hat an der gleichen Stelle der demokratischen Legitimation keinen eigenständigen Platz zugewiesen, sondern sie als Spielart der charismatisch oder der rational-legal legitimierten Herrschaftsformen behandelt (Weber 1956, 155-157, 172174). In der zweiten Hälfte dieses Jahrhunderts hat sich freilich der demokratische Legitimationsglauben gegen alle Konkurrenten durchgesetzt und in der politiktheoretischen Diskussion eine uneingeschränkte Monopolstellung erreicht .[4] Wenn also die Europäische Union (und dann erst recht alle anderen supranationalen Institutionen) an einem dauerhaft oder jedenfalls vorerst unaufhebbaren Demokratiedefizit litten, dann wäre diese Feststellung das normative Todesurteil für alle Hoffnungen auf die Wiedergewinnung der Chance einer demokratischen und sozialen Zivilisierung des Kapitalismus auf der europäischen Ebene.

Aber auf welche Weise kann Herrschaft durch Demokratie legitimiert werden? Die knappste Anwort liefert Abraham Lincolns Trias des government of the people, by the people and for the people. In der nationalstaatlichen Demokratie kann das erste dieser Elemente - ein als politisches Gemeinwesen schon konstituiertes "Staatsvolk" als gegeben vorausgesetzt werden, so daß sich die demokratietheoretische Diskussion auf die Frage konzentriert, unter welchen Bedingungen die beiden anderen Voraussetzungen als erfüllt angesehen werden können. Sie entsprechen der analytischen Unterscheidung zwischen input-orientierten und output-orienten Versionen des demokratischen Legitimationsglaubens. In real-existierenden und funktionsfähigen Demokratien gelten beide Begründungen nebeneinander, und sie verstärken sich wechselseitig. Dennoch folgen sie einer jeweils eigenen Logik, die separat expliziert werden kann, und die in real existierenden demokratischen Systemen auch in unterschiedlichem Maße akzentuiert wird.

\subsection{Government by the People}

Ich beginne mit der input-orientierten Logik. Ihr zufolge gelten politische Entscheidungen als legitim, wenn und weil sie auf die Zustimmung des "Demos" zurückgeführt werden können. Den dazugehörigen Legitimationsglauben hat Carl Schmitt $(1983,229)$ auf die einprägsame Formel gebracht: "Was das Volk will, ist 
eben deswegen gut, weil es (das) will". Aber welche guten Gründe lassen sich dafür anführen? Mit der römischen Maxime volenti non fit iniuria läßt sich vielleicht die zwangsweise Durchsetzung einhelliger Entscheidungen legitimieren, [5] aber sobald man von der einstimmigen zur Mehrheitsentscheidung übergeht, fehlt es ja an der individuellen Zustimmung der Dissidenten. Die Herrschaft einer feindseligen Mehrheit kann - dazu braucht man nicht erst an die Goldhagen-Thesen oder an das ehemalige Jugoslawien zu erinnern - für die Minderheit tödlich sein. Aber selbst wenn nicht feindselige, sondern nur egoistisch-rationale Orientierungen unterstellt werden, zeigt die analytische Demokratietheorie, daß die Mehrheitsregel nur unter sehr restriktiven Annahmen nicht zu völlig irrationalen, normativ keineswegs vertretbaren Ergebnissen führt (Riker 1982).6] Normativ zwingend (und darauf kommt es ja für die Begründung einer Gehorsamspflicht an) erscheint eine ausschließlich input-orientierte Legitimation von Mehrheitsentscheidungen jedenfalls nur, wenn man explizit oder stillschweigend entweder von einem organizistischen Volksbegriff ausgeht oder homogene Präferenzen der Staatsbürger unterstellt. Organizistische oder kollektivistische Varianten der Demokratietheorie gehen davon aus, daß Willensträger und Handlungseinheit der Politik nicht die individuellen Mitglieder des Gemeinwesens seien, sondern die wie immer definierte kollektive Identität - die Klasse, die Bewegung, die Partei oder eben "das Volk", dem ein einheitlicher Wille oder jedenfalls ein einheitliches Interesse zugeschrieben wird. Graf Kielmansegg (1977) hat gezeigt, wie sehr auch das Konzept der Volkssouveränität diesem kollektivistisch-identitären Denken verhaftet ist. Vom Standpunkt des normativen Individualismus aus könnte jedenfalls auf dieser Basis eine moralische Verpflichtung zum Gehorsam gegenüber Mehrheitsentscheidungen kaum begründet werden.

Eine zweite, jedenfalls im Prinzip individualistische Variante der input-orientierten Demokratietheorie unterstellt objektiv homogene Interessen der Bürger.[7] Wenn diese Bedingung tatsächlich annähernd erfüllt ist - etwa in Situationen einer gemeinsamen äußeren Bedrohung, der Überausbeutung von erschöpfbaren Umweltressourcen (Ostrom 1990) oder der Sicherung und Produktion von anderen "Gemeinschaftsgütern" - dann kann man im Prinzip von der Existenz einer "richtigen", weil für alle Beteiligten objektiv besten Lösung ausgehen.[8] Wird dies aber unterstellt, dann erscheint es auch durchaus plausibel, das (ggf. durch Diskussion aufgeklärte) Mehrheitsvotum als Annäherung an diese richtige Lösung[9] - und damit im Sinne Rousseaus als Manifestation meiner eigenen "wahren" Präferenz - zu behandeln, die mich auch dann bindet, wenn ich irrigerweise anders votiert haben sollte (Manin 1987). In der Normalsituation der Politik freilich, in der die individuellen Lagen und Interessen uneinheitlich sind, und in der die realen Präferenzen legitimerweise differieren, wird auch diese Variante der inputorientierten Legitimation der Mehrheitsherrschaft zur totalitären Ideologie (Talmon 1955; Offe/ Preuß 1991).

Schließt man aber sowohl die kollektivistische Begründung als auch die generelle Unterstellung homogener Interessen aus, dann - so kann man analytisch zeigen (Scharpf 1997b) - läßt sich eine ausschließlich input-orientierte Begründung der Mehrheitsherrschaft nur noch konstruieren, wenn man auf seiten der Individuen in der Ausübung ihrer Bürgerrolle (und auf seiten der Amtsträger) nicht lediglich egoistische Interessen, sondern zumindest partiell[10] auch gemeinwohl-orientierte oder - was das gleiche besagt - solidarische Präferenzen unterstellt. Diese Unterstellung aber erscheint selbst unter den Prämissen des Rational-ChoiceAnsatzes keineswegs von vornherein unrealistisch (Brennan 1989; Kirchgässner 1992). Sie impliziert, daß die Mitglieder des Gemeinwesens zwar je für sich unterschiedliche Interessen haben, aber daß ihre für die politische Entscheidung relevanten Präferenzen nicht nur den eigenen Nutzen, sondern auch den der anderen 
Mitglieder des Gemeinwesens als Argument enthalten. Wird dies vorausgesetzt, dann gibt es in der Theorie auch hier "richtige" Lösungen,[11] denen bei vollständiger Aufklärung alle zustimmen müßten, und dann müßte wiederum die Minderheit das Mehrheitsvotum gegen sich gelten lassen. Freilich setzt diese, auch unter den Kriterien der normativen Individualismus und einigermaßen realitätsnahen Bedingungen tragfähige Begründung input-orientierter Legitimität die vorgängige Existenz einer Gemeinschaft voraus, deren Mitglieder bereit sind, sich wechselseitig solidarische Orientierungen zu unterstellen. Je gravierender die Opfer sind, die durch Mehrheitsentscheidung potentiell abverlangt werden könnten, desto höher die Anforderungen an den "Gemeinsamkeitsglauben" (Weber 1956, 237), der erst diese Risiken als tragbar erscheinen läßt.

Da es sich hier in der Tat um Phänomene auf der Ebene normativ-kognitiver Überzeugungen handelt, braucht man die Existenz solidarischer Gemeinschaften auch keineswegs als vorgegebene und unveränderbare - etwa ethnisch begründete Faktizität zu behandeln (Smith 1992). Ihre Ausbildung kann durch historische Erfahrung, religiöse, kulturelle oder ideologische Überzeugungsarbeit und insofern auch durch Politik beeinflußt und verändert werden (Neuman 1996). Aber dennoch ist es nicht die Politik selbst, sondern erst deren Wirkung im Bewußtsein der Adressaten und in der Kommunikation zwischen diesen, die den legitimierenden "Gemeinsamkeitsglauben" hervorbringen kann. Wo eine solche "Wir-Identität" (Elias 1987) existiert, mag es in der Tat für die Legitimation politischer Entscheidungen auf der lokalen, regionalen und nationalen Ebene völlig ausreichen, daß sie in einem Referendum die Unterstützung der Mehrheit gefunden haben, und unter besonderen Bedingungen wird man auch bestimmten Wahlergebnissen die Qualität einer plebiszitären Legitimation kontrovers diskutierter politischer Entscheidungen zusprechen können.

$\mathrm{Da}$ aber dies alles nicht für die Legitimation von Entscheidungen der Europäischen Union gelten könnte, braucht kaum hervorgehoben zu werden. Hier fehlt es (noch) an der solidarischen Identität der Europäer (Risse 1997) und noch mehr an politischen Kommunikationsprozessen und vermittelnden Strukturen, durch die ein "Volkswille" in effektive Politik übersetzt werden könnte. Aber selbstverständlich stützt sich (schon wegen der prinzipiell begrenzten Kapazität individueller und öffentlicher Aufmerksamkeit für politische Entscheidungen - Scharpf 1970) auch keine nationale Demokratie ausschließlich oder auch nur in erster Linie auf die input-orientierte Legitimation durch den manifestierten Volkswillen. Überall wird diese ergänzt oder so etwa im Falle einer politisch unabhängigen Verfassungsgerichtsbarkeit oder Nationalbank - sogar verdrängt durch output-orientierte Legitimationsgründe, die sich auf die gemeinwohlorientierte "Richtigkeit" von Entscheidungen und die Problemlösungs-Effektivität von Politik beziehen, und die durchaus auch für die europäische Politik Bedeutung haben können. Diesen will ich mich nun zuwenden.

\subsection{Government for the People}

Aus der Output-Perspektive erscheint die demokratische Politik als government for the people - d.h. als Veranstaltung zur kollektiven Bearbeitung jener gemeinsamen Probleme, welche die Einzelnen weder durch individuelles Handeln noch durch die Teilnahme am Markt oder durch freiwillig-gemeinsames Handeln in "zivilgesellschaftlichen" Interaktionsnetzen lösen könnten. Bezugspunkt und Kriterium der Legitimation sind hier also nicht die authentische Expression und unverfälschte Implementation des "Volkswillens", sondern die gemeinsamen Probleme und Interessen der real existierenden Mitglieder des Gemeinwesens. Aber was sind "gemeinsame Interessen"? Wenn man auch hier zunächst von egoistisch-rational orientierten Bürgern ausgeht, dann sind dies zunächst nur die im 
strikten Sinne "verallgemeinerungsfähigen Interessen" an der Produktion reiner Kollektivgüter und das Interesse an pareto-superioren Lösungen, bei denen niemand gegenüber dem Status quo schlechter gestellt wird. Die normative Implikation wäre eine strikte Begrenzung der Staatsfunktionen im Sinne des liberalen Konstitutionalismus (Mueller 1996). Die normativen Restriktionen lassen sich wesentlich erweitern, wenn man auch die Möglichkeit einer Kompensation von Verlusten durch Ausgleichszahlungen und Koppelgeschäfte einbezieht. Damit erstreckt sich der Bereich legitimierbaren kollektiven Handelns auf die gegenüber dem Pareto-Kriterium viel größere Gesamtmenge aller dem Kaldor-Kriterium entsprechenden Lösungen, welche (im Vergleich zu dem bei Nichthandeln erwartbaren Zustand) die Gesamtwohlfahrt steigern, und bei denen Gewinne und Verluste nach dem Gerechtigkeitskriterium des Äquivalententauschs ausgeglichen werden (Scharpf 1992; 1997b).[12]

Lösungen freilich, die eine Umverteilung nach anderen Gerechtigkeitskriterien als dem der Äquivalenz (also etwa nach Kriterien der Bedürftigkeit oder der Gleichheit Deutsch 1975) implizieren, wären unter der Annahme egoistisch-rationaler Orientierungen der Bürger weiterhin nicht zu legitimieren. Sollen sie zum Gegenstand kollektiv-verbindlicher Entscheidung gemacht werden, so muß man also auch aus der Output-Perspektive wiederum solidarische Orientierungen voraussetzen - denn warum sonst sollte ich mich bei der politischen Verfolgung meiner Interessen auf Gerechtigkeitskriterien einlassen, deren Anwendung meine eigenen Interessen schädigen könnte? In dem Maße also, wie solidarische Umverteilung angestrebt wird, beschränkt sich auch der potentielle Geltungsbereich output-orientierter Legitimation wieder auf präexistente Gemeinwesen, in denen eine "kommunitaristische" Bürgergesinnung vorausgesetzt werden kann.

Aus alldem folgt, daß auch die Output-Legitimation auf eine Wir-Identität angewiesen ist, die mindestens bestimmt, wer dazu gehört und wessen Interessen deshalb berücksichtigt werden müssen. Es folgt ferner, daß die Anforderungen an die Bindekraft dieser Wir-Identität in dem Maße steigen, wie durch kollektiv verbindliche Entscheidungen gravierende Opfer oder die Bereitschaft zu solidarischer Umverteilung abgefordert werden können. Umgekehrt bedeutet dies dann auch, daß politische Einheiten mit schwacher Wir-Identität - also beispielsweise die Europäische Union, aber auch ethnisch, religiös oder ideologisch tief gespaltene Nationalstaaten - nur über eine begrenzte Legitimation verfügen, welche den politischen Handlungsspielraum auf Lösungen beschränkt, die dem Pareto-Kriterium entsprechen, und die solidarische Umverteilung ausschließen. Aus der OutputPerspektive erscheint deshalb die Wir-Identiät als eine Variable, deren unterschiedliche Ausprägungen mit ihren unterschiedlichen Implikationen ohne Rückgriff auf den Alles-oder-Nichts-Begriff der "Volkssouveränität" analysiert werden können. Output-Legitimation ist deshalb auch kompatibel mit der Vorstellung multipler und abgestufter Formen der Vergemeinschaftung für jeweils unterschiedliche Zwecke und mit jeweils unterschiedlichen Verpflichtungen. Eben diese Flexibilität erlaubt es, demokratische Legitimität auch oberhalb des Nationalstaats für möglich zu halten.

Damit freilich sind erst die Kriterien und Grenzen der Output-Legitimation benannt, aber noch nicht die Bedingungen ihrer Möglichkeit. Da hier definitionsgemäß weder bei den Mitgliedern des Gemeinwesens noch bei den Inhabern der Staatsgewalt von vornherein identische oder solidarische Interessen unterstellt werden können, muß das Problemlösungspotential der Politik immer zugleich auch als ein Ausbeutungsund Unterdrückungspotential verstanden werden. Output-orientierte Legitimation erfordert deshalb institutionelle Vorkehrungen, die einerseits den Mißbrauch des Herrschaftspotentials ausschließen, und andererseits dessen effektive Verwendung zugunsten der gemeinsamen Interessen ermöglichen sollen. Da überdies auch 
gemeinschaftsdienliche Maßnahmen die Mitglieder in höchst ungleichem Maße begünstigen oder belasten können, sind notwendigerweise auch Regeln zur Sicherung der Verteilungsgerechtigkeit erforderlich.

Charakteristisch für output-orientierte Legitimationsdiskurse ist deshalb ein primäres Interesse für die Konstruktion und Evaluation institutioneller Vorkehrungen, welche gemeinwohlorientiertes politisches Handeln ermöglichen und Mißbrauch ausschließen sollen. Sie erst, und nicht der unhinterfragte "Volkswille", begründen die Legitimät des politischen Systems und damit die Gehorsamspflicht gegenüber regelgerecht zustande gekommenen kollektiven Entscheidungen. Die Argumente dafür haben in den Federalist Papers (Cooke 1961) ihren klassischen Ausdruck gefunden, aber die tatsächliche Formenvielfalt der diesem doppelten Zweck dienenden Institutionen geht über die Checks and Balances der amerikanischen Präsidialdemokratie weit hinaus. Sie schließt die Parteienkonkurrenz im britischen Westminster-System ebenso ein wie die Allparteienverhandlungen in der schweizerischen Konkordanzdemokratie oder die vertikale Kompetenzverflechtung im deutschen Föderalismus. Hinzu kommen fast überall rechtlich garantierte Grundrechte, oft auch eine unabhängige Verfassungsgerichtsbarkeit, und manchmal eine unabhängige Zentralbank, rechtlich gesicherte Regelungskompetenzen der Sozialpartner oder der Kirchen, Garantien der kommunalen Selbstverwaltung und der staatlich gestützten Selbstorganisation mancher Professionen und Funktionsbereiche. Die schier unübersehbare Formenvielfalt machtschaffender und machtbegrenzender und insoweit legitimierender institutioneller Arrangements muß freilich auf abstraktere Mechanismen zurückgeführt werden, die eine vergleichende Betrachtung und Beurteilung unterschiedlicher Lösungen in unterschiedlichen Systemen ermöglichen. Hier hat George Tsebelis mit seiner Klassifikation politischer Institutionen nach der Zahl und Art ihrer "veto players" (Tsebelis 1995; Tsebelis/ Money 1997) einen wichtigen theoretischen Durchbruch erzielt, der jedoch für die hier zu erörternden Fragen eine zu radikale Vereinfachung darstellt. Statt dessen unterscheide ich drei[13] jeweils eigenständigen Mechanismen[14] zur Sicherung gemeinwohlorientierter politischer Entscheidungen, nämlich

- institutionalisierte Mechanismen, deren Wirkung auf der direkten oder indirekten Abhängigkeit der Regierenden von den Regierten beruht, und die deshalb entweder die "demokratischen" Inputs selbst - also Volksabstimmungen, allgemeine Wahlen und die öffentliche Meinung - oder (und vor allem) die antizipierende Reaktion der Machthaber auf diese Inputs zur Disziplinierung der Herrschaftsausübung einsetzen; oder

- Mechanismen, deren Wirkung auf der Institutionalisierung von unterschiedlich legitimierten Vetopositionen beruht, und die Verhandlungen zwischen den Positionsinhabern zur Annäherung an gemeinwohldienliche politische Lösungen einsetzen; oder schließlich

- Mechanismen, deren Wirkung auf der Orientierung an konsensfähigen Kriterien des "objektiv" Richtigen und Gebotenen beruht, und die deshalb Entscheidungen auf politisch unabhängige und fachlich kompetente Gremien (beispielsweise Gerichte oder Zentralbanken) verlagern, die in erster Linie durch professionelle Selbstkontrollen diszipliniert werden sollen.

Die erste Gruppe von im engeren Sinne "demokratischen" Mechanismen werden hier nicht in ihrer input-orientierten Bedeutung als Ausdruck des "Volkswillens" betrachtet, sondern sie erscheinen als instrumentelle Vorkehrungen zur Sicherung der Gemeinwohlorientierung der Inhaber der Staatsgewalt. Faktisch beruht ihre legitimierende Wirkung in erster Linie auf Carl J. Friedrichs $(1937,16)$ berühmtem Gesetz der "antizipierenden Reaktion" in Verbindung mit der Tatsache, daß die 
politische Kommunikation unter den handelnden Akteuren, und die Kommunikation zwischen diesen und ihren Wählern, unter der potentiellen Aufmerksamkeit einer politischen Öffentlichkeit stattfinden muß, und daß über öffentliche Angelegenheiten in der Öffentlichkeit mit Bezug auf das gemeinsame Interesse diskutiert und gestritten werden kann. Wer öffentlich kritisiert oder verteidigt, muß dies mit öffentlich vorzeigbaren Argumenten tun - und wenn das Argument sich auf Gegenstände von allgemeiner Bedeutung richtet, dann sind nur Argumente vorzeigbar, die sich auf die Förderung oder Verletzung des allgemeinen Interesses oder auf Maßstäbe der Verteilungsgerechtigkeit beziehen (Elster 1983, 35-36; 1986; 1991; Habermas 1973; 1992, Kap. VIII ). 15] Die öffentliche Argumentation verstärkt also das Gewicht gemeinwohlorientierter Kriterien im Bewußtsein der Wähler und damit zugleich das Risiko für die Regierenden, daß gemeinwohlschädliche Politik sich tatsächlich auf Wahlergebnisse auswirken könnte (Scharpf 1997b, 183-193).

Das heißt keineswegs, daß alle oder wenigstens alle wichtigen politischen Entscheidungen aus öffentlichen Diskursen hervorgehen müßten. Öffentliche Aufmerksamkeit ist eine extrem knappe Ressource. Es genügt, daß die Regierenden ihre Politik unter Ungewißheit wählen müssen - zu einem Zeitpunkt, an dem sie noch nicht wissen können, welche Themen die Opposition politisieren, welche die Medien aufgreifen, und für welche davon die Wähler sich am Wahltag noch interessieren werden. Wenn aber überhaupt die Möglichkeit besteht, daß gemeinwohlorientierte Wechselwähler den Ausgang der Wahl entscheiden könnten, und wenn deren Entscheidung durch öffentliche politische Diskussion beeinflußt werden kann, dann haben Regierungen und Parlamentsmehrheiten ein sehr starkes Motiv, Politiken zu wählen, die auch in kontroverser öffentlicher Diskussion mit guten GemeinwohlArgumenten verteidigt werden können (Scharpf 1997b, 183-188). Das schließt ideologischen Bias und Fehlurteile gewiß nicht aus, aber die absichtsvolle Vereitelung gemeinwohldienlicher Lösungen ist in der Politik demokratischer Verfassungsstaaten doch eher die Ausnahme.

Im Prinzip gilt dies auch für die nicht von Wahlen abhängige Expertokratie der Verfassungsgerichtsbarkeit oder der Zentralbank, deren Unabhängigkeit in einem möglichen Konflikt mit Regierung und Parlament am Ende auch nur durch die antizipierte Reaktion der Öffentlichkeit und der Wähler gesichert ist. So zwang die öffentliche Meinung zwar den amerikanischen Präsidenten Franklin Delano Roosevelt im Verfassungskonflikt von 1937 zum Verzicht auf die angekündigte Änderung des Richterwahlgesetzes - aber eben deshalb mußte der Supreme Court schließlich doch die Verfassungsmäßigkeit der New-Deal-Gesetzgebung bejahen (Schwartz 1957; Ehmke 1961). Aus dem gleichen Grund konnte die Deutsche Bundesbank sich zwar bei der Bewertung der Goldbestände gegen Finanzminister Waigel durchsetzen, nicht aber gegen den Kanzler, als es 1990 um die deutsche Währungsunion ging. Genereller formuliert: Gerade formell unabhängige Einrichtungen sind - jedenfalls dann, wenn sie in Konflikt mit den politisch legitimierten Instanzen geraten - in ganz besonderem Maße gezwungen, ihre Position mit Gemeinwohl-Argumenten zu begründen, die in der öffentlichen Meinung Unterstützung finden können. Ihre Unabhängigkeit läßt sich nur so lange verteidigen, wie ihre Politik - in den Worten eines der großen amerikanischen Richter - "the sober second thought of the community" repräsentiert (Bickel 1962, 26).

Von Bedeutung ist die Einbettung in den Kontext öffentlicher politischer Diskussion aber auch für die Verhandlungssysteme, in denen ein großer Teil unserer Politikproduktion tatsächlich stattfindet. Hier moniert die input-orientierte Kritik, daß Verhandlungen - zwischen Koalitionsparteien, zwischen Bund und Ländern, zwischen Regierung und Sozialpartnern oder auch zwischen dem amerikanischen Präsidenten und dem Kongreß oder zwischen dem Präsidenten und dem 
Ministerpräsidenten in der französischen cohabitation - die unmittelbare Verantwortung gegenüber dem eigenen Parlament, den eigenen Wählern oder den eigenen Mitgliedern schwächen müssen. Aus der Output-Perspektive freilich wäre diese Kritik nur dann relevant, wenn durch einseitiges Handeln für die Mitglieder bessere Ergebnisse erzielt werden könnten.

Wenn aber brauchbare Lösungen nicht im jeweils eigenen Kompetenzbereich und mit den eigenen Ressourcen der beteiligten Organisationen oder Gruppen erreicht werden können, sondern nur im Zusammenwirken formell eigenständiger Einheiten, dann können auch die artikulierten Präferenzen der Mitglieder einer jeden der beteiligten Einheiten nur in eingeschränktem Maße als Kriterium dienen. Solange keine Seite einer Vereinbarung zustimmen muß, bei der sie sich schlechter stellt als bei einem Scheitern der Verhandlungen, sind Verhandlungsergebnisse in der OutputPerspektive im Prinzip legitimiert. Selbstverständlich kann es in jeder der beteiligten Einheiten Streit darüber geben, ob bei einer härteren oder geschickteren Verhandlungstaktik für die eigene Seite vielleicht noch mehr hätte herausgeholt werden können. Aber nichts spricht für die Vermutung, daß die Ergebnisse für alle beteiligten Gruppen - und damit für das Gemeinwohl - verbessert werden könnten, wenn alle Verhandlungsführer durch imperatives Mandat oder strikte parlamentarische Kontrollen an die Inputs ihrer jeweiligen Auftraggeber gebunden würden.

Die charakteristische Schwäche von Verhandlungssystemen liegt also nicht in der Abkoppelung von demokratischen Kontrollen, sondern in der Gefahr, daß die Parteien bei der hartnäckigen Verfolgung der von ihnen zu vertretenden Partialinteressen das Scheitern der Verhandlungen in Kauf nehmen und so gemeinwohldienliche Lösungen überhaupt vereiteln könnten. Gerade gegen diese Gefahr aber bietet die Einbettung der Verhandlungen in eine die partiellen Mitgliedschaften umgreifende politische Öffentlichkeit einen gewissen Schutz. Jeder der Beteiligten muß hier ja - wie sich gerade derzeit in der deutschen Innenpolitik wieder zeigt - mit dem Risiko rechnen, daß negative Reaktionen der Öffentlichkeit auf allfällige Verhandlungsblockaden auch bei den eigenen Wählern ihre Wirkung entfalten könnten.

\section{Europäische Politik: Begrenzte Legitimität}

Was aber folgt nun aus diesen Überlegungen für die demokratische Legitimation der europäischen Politik? Im Nationalstaat, so ist zu erinnern, stehen gut begründbare input-orientierte und output-orientierte Legitimationsargumente nebeneinander und verstärken sich wechselseitig. Entscheidungen, die in plausibler Weise auf die Willensäußerung einer Wählermehrheit oder einer mit klarem Mandat ausgestatteten parlamentarischen Mehrheit zurückgeführt werden können, haben starke Legitimationsgründe für sich - und dies um so mehr, weil ja die vorhandenen (und aus output-orientierter Sicht notwendigen) Vorkehrungen gegenüber möglichen Machtmißbrauch zusätzliche Sicherheit bieten. Umgekehrt deckt die OutputLegitimation Bereiche des Staatshandelns, für die ein plausibler Bezug auf den aktuellen Wählerwillen keineswegs behauptet werden könnte, aber die insoweit legitimierenden Argumente werden ergänzt und verstärkt durch die Einbettung aller Interaktionen in den Kontext einer politischen Öffentlichkeit, die als fleet in being nicht nur gegenüber den von Wahlen abhängigen Akteuren wirkt, sondern auch gegenüber Verhandlungssystemen und unabhängigen Institutionen, die potentiell auf öffentlichen politischen Druck reagieren müßten.

\subsection{Fehlende Input-Legitimation}

Für die europäische Politik dagegen fehlt es an den Grundlagen der input-orientierten Legitimation. Dies liegt, ich brauche es kaum noch zu betonen, nicht in erster Linie an der unvollständigen Kompetenzausstattung des direkt gewählten Europäischen Parlaments. Auch wenn dieses die vollen legislativen und politischen Kompetenzen 
eines nationalen Parlaments hätte, könnten seine Entscheidungen gewiß nicht allein mit dem Hinweis auf das Mehrheitsprinzip legitimiert werden. Das dafür ausschlaggebende Argument wurde von Joseph Weiler in der rhetorischen Frage auf den Punkt gebracht hat, was denn nach einem etwaigen Anschluß an Deutschland die Dänen dazu bringen könnte, ein Mehrheitsvotum im Bundestag als moralisch verpflichtend anzusehen (Weiler 1996). Damit soll gesagt sein, daß den Bürgern der Europäischen Union jedenfalls bisher jene solidarische Wir-Identität und jene das Gemeinwesen umfassende politische Öffentlichkeit fehlt, die erst das Mehrheitsvotum als solches - nach der Maxime "the people can do no wrong" legitimieren könnte (Niedermayer/ Sinnot 1995). Deshalb gehen auch alle Vorschläge zur Überwindung des "europäischen Demokratiedefizits" durch die Einführung von europaweiten Plebisziten (Grande 1996; Zürn 1996) am Problem vorbei.

$\mathrm{Da}$ sich das bald ändern könnte, ist schon deshalb unwahrscheinlich, weil ja nirgendwo identitätsbildende Anstrengungen von der Art der italienischen und deutschen Nationalbewegungen des letzten Jahrhunderts oder der zionistischen Bewegung am Beginn dieses Jahrhunderts am Werk zu sein scheinen (Gellner 1983; Smith 1992), und weil Sprachbarrieren auch den Austausch zwischen den nationalen Kommunikationssystemen eng beschränken. Zwar schwächt die ökonomische und institutionelle Integration Europas möglicherweise die Bindekraft des Nationalstaats aber den Gewinn davon haben bisher eher die subnationalen - schottischen, katalanischen, flandrischen, padanischen und vielleicht bayerischen - Identitäten und nicht eine neue europäische Identität (von der man ja noch nicht einmal wüßte, wer denn am Ende dazugehören soll).

Kurz, die input-orientierte Legitimation, und damit die prinzipielle Geltung des Mehrheitsprinzips steht jedenfalls bisher für die Herrschaftsausübung oberhalb des Nationalstaats nicht zur Verfügung. Wer also, wie etwa das Bundesverfassungsgericht in seinem Maastricht-Urteil, die Input-Dimension des Demokratiebegriffs stark akzentuiert, und deshalb gezwungen ist, einen stark integrierten - organizistischen, homogenen oder solidarischen - Demos-Begriff vorauszusetzen, der muß dann folgerichtig nicht nur die demokratische Legitimation der Europäischen Union in ihrer gegenwärtigen Gestalt verneinen, sondern auch die Chancen einer künftigen Überwindung des europäischen Demokratiedefizits skeptisch einschätzen (Kielmansegg 1996). Aus der hier entwickelten Perspektive wäre damit freilich noch nicht das letzte Wort über die Legitimität der europäischen Politik gesprochen.

\subsection{Schwache Output-Legitimation}

Allerdings muß die Legitimation der europäischen Politik dann ausschließlich mit output-orientierten Argumenten begründet werden, und diese können überdies auch nicht auf die im engeren Sinne "demokratischen"[16] Kontrollmechanismen Wahlen, Abstimmungen und Öffentlichkeit - verweisen. Damit fehlt nicht nur die Möglichkeit der direkten oder indirekten Legitimation majoritärer Entscheidungen, sondern auch die legitimationsverstärkende Einbettung der verbleibenden Mechanismen in den Kontext einer potentiell sanktionskräftigen politischen Öffentlichkeit. Die auf sich allein gestellte Output-Legitimation ist also schwächer und weniger belastbar, als sie es in Kombination mit input-orientierten Mechanismen wäre - aber sie ist darum doch keineswegs bedeutungslos.

\subsubsection{Negative Integration durch autonomes Recht}

Am wenigsten umstritten erschien bis vor kurzem die Legitimität der sogenannten "negativen Integration", also der unmittelbar auf den Vertragstext gestützten Interventionen der Kommission und des Europäischen Gerichtshofs gegen nationale Handelshindernisse und wettbewerbsverzerrende Maßnahmen (Scharpf 1996). Sie wurde in der deutschen juristischen Diskussion zunächst mit der "Zweckverbandstheorie" (Ipsen 1972) unmittelbar aus den historischen Intentionen der vertragschließenden Regierungen und ratifizierenden Parlamente abgeleitet. In 
dem Maße freilich, wie die negative Integration in der Praxis der Kommission und des Gerichts über die ursprünglichen Intentionen der Vertragsparteien hinausging, radikalisierte sich auch das Legitimationsargument zur - insbesondere von deutschen Wettbewerbsrechtlern vorangetriebenen - Konstruktion einer quasi-naturrechtlich begründeten europäischen "Wirtschaftsverfassung" (Behrens 1994). Rechtstechnisch abgestützt durch die Doktrinen der unmittelbaren Geltung und des Vorrangs der Verträge vor allem nationalen Recht, und inhaltlich abgeleitet aus der zu Grundrechten uminterpretierten vertraglichen Verpflichtung zur Schaffung eines Binnenmarktes, "der durch die Beseitigung der Hindernisse für den freien Waren-, Personen-, Dienstleistungs- und Kapitalverkehr zwischen den Mitgliedstaaten gekennzeichnet ist" (Artikel 2 e EGV), sollen nach dieser Lesart die Grundsätze einer radikal marktliberalen Wirtschaftsverfassung nun sogar die Mitgliedstaaten in ihrer Rolle als "Herren der Verträge" binden (Mestmäcker 1994).

Während hier die im Prozeß der europäischen Integration möglich gewordene Ablösung des autonomen Rechts von der Politik gefeiert wird, betont eine zweite Argumentationslinie die eigenständige Legitimation von ausschließlich an Kriterien der Effizienz orientierten unabhängigen Regulierungskommissionen und Notenbanken (Ladeur 1992; Majone 1996). Auf der europäischen Ebene soll dies beispielsweise für die wettbewerbsrechtliche Regulierungstätigkeit der Kommission gelten - also für den Bereich, auf den sich auch die Literatur zur Wirtschaftsverfassung konzentriert. Genau das gleiche Argument wird aber auch vom Bundesverfassungsgericht in seinem Maastricht-Urteil an der Stelle benutzt, wo es ganz im Gegensatz zu seinem ansonsten extrem input-orientierten

Demokratieverständnis - die politische Unabhängigkeit der künftigen europäischen Zentralbank deshalb für legitim hält, weil die "Sicherung des in eine Währung gesetzten Einlösungsvertrauens" auf diese Weise besser gewährleistet sei als durch "Hoheitsorgane, die ihrerseits ... auf die kurzfristige Zustimmung politischer Kräfte angewiesen sind."[17]

Aber was immer solche Begründungen im Nationalstaat zur Legitimation der Expertokratie beitragen mögen - im europäischen Kontext sind sie weniger überzeugend, weil die Möglichkeiten der demokratisch-politischen Revision einer sich vom Gemeinwohlverständnis der Gemeinschaft entfernenden Entscheidungspraxis viel schwächer ausgestaltet sind. Das Bundesbankgesetz wäre, wenn Herr Waigel seine parlamentarischen Truppen hätte zusammenhalten können, mit einfacher Mehrheit zu ändern gewesen, aber zur Änderung der MaastrichtArtikel, welche die Zusammensetzung und die Kompetenzen der künftigen Europäischen Zentralbank im Detail regeln (Art. 105 - Art. 109 d, EGV), bedürfte es einer einstimmigen Vertragsänderung, die von allen nationalen Parlamenten zu ratifizieren wäre. Das gleiche gilt für das Wettbewerbsrecht, das im Nationalstaat zur Disposition des einfachen Gesetzgebers steht, in der Europäischen Union aber Verfassungsqualität gewinnen konnte - mit der Folge, daß auch hier Entscheidungen des EuGH nur durch einstimmige und ratifikationsbedürftige Vertragsänderungen formell korrigiert werden könnten.

Dies gibt dem Europarecht zwar eine hohe Durchschlagskraft solange seine Expansion von den nationalen Gerichten gestützt wird,[18] aber der Verzicht auf politische Legitimation impliziert auch höhere Verwundbarkeit im Falle eines politischen Konflikts. Das Risiko solcher Konflikte hat zugenommen, seitdem die Kommission seit Mitte der achtziger Jahre die Anwendung des europäischen Wettbewerbsrechts über die marktwirtschaftlich verfaßten Sektoren hinaus auf "staatsnahe Sektoren" (Mayntz/ Scharpf 1995) ausgeweitet hat, die in der nationalen Praxis bis dahin als "Ausnahmebereiche" der Geltung des Kartellrechts entzogen waren. Während die Liberalisierung der Telekommunikation mit der wenigstens stillschweigenden Unterstützung der meisten Regierungen vorangetrieben werden 
konnte, gab es bei der Liberalisierung des Luftverkehrs, des Straßengüterverkehrs und jetzt der Elektrizitätsversorgung erhebliche Widerstände in einzelnen oder mehreren Mitgliedstaaten (Schmidt 1997).

Sie müssen sich verstärken, je mehr die Anwendung der ökonomisch-juristischen Syllogismen des "unverfälschten Wettbewerbs" sich dem Kernbereich der öffentlich erbrachten, öffentlich finanzierten oder öffentlich regulierten Infrastruktur- und Dienstleistungen nähert, die in Deutschland als "Daseinsvorsorge" und in Frankreich als "service public" zu den legitimierenden Aufgaben des modernen Staates gehört haben. Es ist deshalb interessant zu beobachten, daß nun in der Praxis der Kommission, in den Entscheidungen des Gerichts und in besonderer Deutlichkeit in Beschlüssen auf dem Amsterdamer Gipfel zumindest retardierende Elemente zu beobachten sind. Die Liberalisierung des Energiemarktes läßt national unterschiedliche Lösungen zu, das Postmonopol wird nicht generell dem Wettbewerb geopfert, die deutschen öffentlichen Banken werden in ihrer Infrastrukturfunktion anerkannt, das öffentlich-rechtliche Fernsehen soll in seiner Sonderstellung respektiert werden. Kurz: die scheinbar unaufhaltsame Expansion der auf Marktöffnung, Liberalisierung, Deregulierung und Privatisierung gerichteten europäischen Wettbewerbspolitik scheint nun einer Abwägung zwischen Marktfreiheit und öffentlichen Aufgaben Platz zu machen, die den offenen Legitimationskonflikt vermeiden könnte (Scharpf 1997c).

\subsubsection{Positive Integration durch Verhandlungen}

Die institutionellen Bedingungen, welche die negative Integration begünstigen, stehen jedoch für Maßnahmen der "positiven Integration", das heißt für marktbeschränkende und marktkorrigierende Regelungen auf der europäischen Ebene, die den Verlust an Regelungskompetenz ausgleichen können, der auf der nationalen Ebene infolge der ökonomischen Integration eingetreten ist, keineswegs zur Verfügung. Solche Regelungen können nicht unter Ausschluß der Politik als bloße Interpretation der Verträge legitimiert und allein von der Kommission und dem Gericht vorangetrieben werden. Da sie aus den zuvor genannten Gründen auch nicht majoritär legitimiert werden könnten, bleibt nur die Legitimation durch Verhandlungen. Diese ist, ich sagte es schon, besser als ihr Ruf in der demokratietheoretischen Diskussion, jedenfalls wenn man berücksichtigt, daß nach der Logik von Kollektivverhandlungen input-orientierte Kriterien ohnehin nicht relevant sein können. Unter normativen Gesichtspunkten kommt es dabei allein darauf an, ob die effektiv betroffenen Interessen in den Verhandlungen vertreten sind, und ob die Transaktionskosten niedrig genug gehalten werden können, um die möglichen Wohlfahrtsgewinne von Verhandlungslösungen auch tatsächlich zu realisieren (Scharpf 1992; 1997b).

Zumindest unter dem ersten Kriterium erscheinen die Verhandlungssysteme der EUPolitik als annähernd ideale Konstruktion. Unmittelbar beteiligt sind als Inhaber formaler Vetopositionen die jeweils zuständigen Generaldirektionen, ohne deren Initiative Prozesse der Politikformulierung gar nicht in Gang kommen; die Mehrheit der Kommissare, die einer Kommissionsinitiative zustimmen müssen; die jeweils zuständigen nationalen Ministerien, die im Ministerrat eine vorgeschlagene Richtlinie entweder einstimmig oder mit qualifizierter Mehrheit billigen müssen, und in einer zunehmenden Zahl von Fällen auch die Mehrheit im Europäischen Parlament. Repräsentiert sind also mindestens alle jene Interessen, die in irgendeinem Mitgliedsland Einfluß auf das zuständige Ressort haben; zusätzlich die vielfältigen Expertengruppen und Interessenten, die von der zuständigen Generaldirektion im Vorfeld von Ratsentscheidungen und danach in den die Umsetzung begleitenden Gremien der Comitologie konsultiert werden; und schließlich auch die "diffusen" Interessen des Umwelt- und Verbraucherschutzes, deren sich das Europäische Parlament mit besonderem Engagement angenommen hat (Pollack 1997). Kurz: Das 
"Interessenberücksichtigungspotential" (um einen Modebegriff der deutschen Politologie der siebziger Jahre zu zitieren) der europäischen Verhandlungssysteme ist jedenfalls nicht geringer, sondern vermutlich höher als das der nationalstaatlichen Politik.

Freilich gilt dies nur für die Verhinderung von interessenschädigender Politik. Wenn es dagegen um positive Aktion geht, dann ist ein so komplexes Verhandlungssystem wie das europäische leicht zu hemmen oder auch völlig zu blockieren. Dies ist nichts Neues (Scharpf 1985). Aus normativer Perspektive führt das allerdings zu einer eindeutigen und vielleicht überraschenden Schlußfolgerung: Im Vergleich zum Nationalstaat bieten die institutionellen Strukturen der Europäischen Union höhere Gewähr dafür, daß die Maßnahmen der positiven Integration, die tatsächlich beschlossen werden können, den Kriterien der Output-Legitimation in vollem Umfange gerecht werden. Die Schwäche der europäischen Politik liegt also nicht in der mangelnden Legitimität, dessen was getan wird, sondern in ihrer geringen Handlungsfähigkeit oder, genauer gesagt, in der Diskrepanz zwischen den an die europäische Politik adressierten Erwartungen und ihrer tatsächlichen Problemlösungsfähigkeit.

An dieser delegitimierenden Diskrepanz haben viele mitgewirkt: nationale Politiker, die bei jeder Gelegenheit nach "europäischen Lösungen" rufen, ebenso wie visionäre europäische Politiker, die in immer neuen Anläufen den "großen Sprung nach vorn" versucht haben, der unter den gegebenen Verhältnissen doch nicht gelingen kann. Die Europäische Union, so kann man abschließend sagen, gewinnt an Legitimation in dem Maße, in dem alle Beteiligten sich darauf einstellen, von Brüssel nur Lösungen für Probleme zu erwarten, die im Prinzip einvernehmlich gelöst werden können. Diesen Bereich kann man einigermaßen präzise umschreiben; er umfaßt wichtige Bereiche nützlicher Politik, die auf der nationalen Ebene nicht mehr und auf der europäischen in völlig befriedigender Weise bearbeitet werden (Scharpf 1997). Hierzu gehören nicht nur die Außenhandelspolitik und die Politiken der negativen Integration, insbesondere die Wettbewerbspolitik, sondern auch das weite Feld der einheitlichen Produktnormen, die erst den größeren europäischen Markt möglich machen. Das Potential der gemeinsamen europäischen Politik ist auch noch keineswegs ausgeschöpft; es könnte, wie ich an anderer Stelle gezeigt habe, durch die systematische Suche nach zugleich problemlösenden und konfliktminimierenden Instrumenten der differenzierenden Regulierung, die auf die unterschiedlichen Interessen der einzelnen Mitgliedstaaten Rücksicht nehmen, noch erheblich ausgeweitet werden (Scharpf 1996; 1997).

Was freilich auf der europäischen Ebene vorderhand nicht erreichbar ist, das sind Maßnahmen der positiven Integration in Bereichen, in denen entweder gravierende Interessenkonflikte zwischen den ökonomisch hochentwickelten und den weniger entwickelten Mitgliedstaaten zu erwarten sind, oder in denen angesichts tiefgreifender Unterschiede zwischen den nationalen Institutionen einheitliche europäische Lösungen exorbitante Veränderungskosten hätten. Unglücklicherweise treffen diese Bedingungen gerade in den Politikfeldern zu, in denen auf der nationalen Ebene die negativen Folgen der ökonomischen Integration am stärksten spürbar werden - nämlich in der Beschäftigungs- und Sozialpolitik (Leibfried/ Pierson 1995; Scharpf 1997). Das schließt nicht aus, daß auch hier Fortschritte erreichbar wären, sofern Lösungen gesucht werden, welche die ökonomischen und institutionellen Interessenkonflikte ausblenden können. Aber im Prinzip bleibt es doch dabei, daß die gravierendsten politischen Folgeprobleme der ökonomischen Integration für die europäischen Sozialstaaten nicht durch europäische Lösungen und erst recht nicht durch globale Vereinbarungen zu deren Gunsten geregelt werden können.

Die Rede vom "europäischen Demokratiedefizit", so kann man diese Argumente 
zusammenfassen, geht also an den Realitäten der europäischen Politik vorbei. Majoritäre Entscheidungen wären in der Tat aus normativen Gründen nicht zu legitimieren - aber sie werden ja auch nicht praktiziert oder auch nur ernsthaft gefordert. Die Verhandlungslösungen dagegen, die in der Union tatsächlich erreicht werden können, lassen sich demokratietheoretisch mindestens so gut legitimieren wie die Ergebnisse der deutschen Politikverflechtung. Die Politikwissenschaft und die politische Diskussion täten deshalb gut daran, statt immer noch einmal das "demokratische Fiasko der Europäischen Union" (Narr 1997) zu beklagen, deren durchaus begrenzte Handlungsfähigkeit - und damit auch die vergleichsweise geringe Virulenz von Legitimationsproblemen - zur Kenntnis zu nehmen und im übrigen die Diskussion über Demokratiedefizite auf der nationale Ebene weiterzuführen, wo die ökonomische Beschränkung bisher vorausgesetzter politischer Gestaltungsmöglichkeiten in der Tat zum Legitimationsproblem werden kann.

\section{Nationalstaatliche Demokratie ohne Omnipotenz}

Die exogene Beschränkung staatlicher Handlungsspielräume wird in erster Linie für die input-orientierten Legitimationskonzepte zum Problem, die demokratische Herrschaft als Ausdruck des Volkswillens und Manifestation der Volkssouveränität definieren. Sie müssen nach ihrer immanenten Logik innerhalb des Gemeinwesens die Kompetenzkompetenz und damit faktisch die Omnipotenz der demokratischen Politik und nach außen ein hohes Maß an Autonomie und jedenfalls rechtliche Handlungsfreiheit voraussetzen (Hindess 1991). Was das Volk will, ist nicht nur per se gut, sondern es muß auch getan werden. Aus dieser Perspektive müssen deshalb sowohl die faktische Beschränkung der staatlichen Politik durch die neuen ExitOptionen der Kapitalanleger, Unternehmen und Konsumenten als auch die rechtlichen Schranken der negativen Integration als ein nicht kompensierbarer Verlust an demokratischer Selbstbestimmung erscheinen.

Aus der output-orientierten Problemlösungsperspektive dagegen werden Omnipotenzvorstellungen schon durch die rechtsstaatlichen, föderalen und prozeduralen Vorkehrungen gegen den Machtmißbrauch ausgeschlossen. Politik erscheint hier als ein institutionalisiertes Verfahren zur Bearbeitung gesellschaftlicher Probleme mit vorgegebenen Mitteln, nach vorgegebenen Regeln und mit vorgegebenen Beschränkungen. Ihre Legitimation gewinnt sie daraus, daß sie unter Beachtung ihrer normativen Beschränkungen und unter den gegebenen Umständen das im Interesse der Bürger Nötige und Mögliche tut. Nicht populistische Willfährigkeit ist hier also gefordert, sondern Problemlösungskompetenz und Ergebnisverantwortung. Gewiß kann und wird es über den einzuschlagenden Weg und über die richtige Verteilung der Lasten politischen Streit geben, und Wahlergebnisse - insbesondere, wenn sie das Mandat einer amtierenden Regierung bestätigen - können durchaus auch als input-orientierte Legitimation einer bestimmten Politik interpretiert werden. Ein wichtigeres Indiz für die faktisch geltenden Kriterien ist jedoch die Tatsache, daß Regierungen typischerweise dann abgewählt werden, wenn der Eindruck sich verbreitet, daß sie das Ende ihrer Problemlösungskompetenz erreicht haben.

Das heißt keineswegs, daß in der demokratischen Politik nur Erfolge honoriert werden: Winston Churchill und seine Regierung haben nach Dünkirchen gewiß nicht an Legitimation verloren, und auch Helmut Schmidt ist aus der ersten Ölpreis-Krise eher gestärkt hervorgegangen. Demokratie erlaubt nicht nur das Segeln vorm Wind bei leichter Brise und strahlender Sonne. Aber wenn das Wetter schlechter wird, dann steigen die Anforderungen an Steuermann und Mannschaft; und dann kann der Eindruck von Hilflosigkeit und Handlungsunfähigkeit angesichts steigenden Problemdrucks nicht nur Regierungen stürzen, sondern auch die Legitimation des 
demokratischen Regierungssystems überhaupt untergraben.

Deshalb sind die gegenwärtigen Krisensymptome steigender Massenarbeitslosigkeit, sich ausbreitender Armut und brüchig werdender sozialer Sicherungssysteme durchaus ernst zu nehmen. Aber es sind zunächst nur Herausforderungen der Politik, nicht Krisen der Demokratie. Die internationalisierte Ökonomie erzeugt Probleme und Gefahren. Aber ebenso wie in der Weltwirtschaftskrise der dreißiger Jahre Länder wie Schweden, Frankreich, Großbritannien und die USA ganz unterschiedliche Lösungen gesucht und gefunden haben, ohne die demokratische Legitimation zu gefährden, so sind auch in der gegenwärtigen Krise unterschiedliche Länder - Großbritannien, Neuseeland, die Niederlande, Dänemark, Schweden - dabei, je eigene politische Antworten auf die neuen Herausforderungen der Globalisierung zu entwickeln. Für die Legitimation des politischen Systems kommt es offenbar auch nicht darauf an, ob alle sozialen Errungenschaften der Nachkriegsjahrzehnte verteidigt werden können, oder ob neue, im öffentlichen Diskurs geprüfte und akzeptierte Konfigurationen von gesellschaftlicher Solidarität und ökonomischer Effizienz erprobt werden. Entscheidend ist, ob das Vertrauen erhalten bleibt, daß im Verhältnis zur drohenden Gefahr und im gemeinsamen Interesse das Mögliche unternommen wird, um Schaden vom Gemeinwesen abzuwenden und die unvermeidbaren Verluste gerecht zu verteilen.

Bleibt dieses Vertrauen erhalten, dann kann auch eine Weltwirtschaftskrise die Demokratie nicht gefährden. Wird es zerstört, dann droht nicht nur die Abwahl von Regierungsparteien, sondern die Erosion politischer Legitimation mit allen Folgen von der zynischen Ausbeutung des Systems bis zum gewaltsamen Protest und von der passiven Abwendung von der demokratischen Politik bis hin zur aggressiven Radikalisierung. Insoweit bleibt Weimar eine Warnung. Für uns als Politikwissenschaftler könnte dies bedeuten, daß ganz oben auf unserer Traktandenliste nicht die Sorge um das europäische Demokratiedefizit stehen sollte, sondern die vergleichende Erforschung der Bedingungen, unter denen jedenfalls manche Länder die Fähigkeit gewinnen, auch unter Krisendruck problembezogene und handlungsrelevante und deshalb legitimationskräftige politische Diskurse zu führen und deren Ergebnisse in effektive Politik umzusetzen.

\section{Literaturverzeichnis}

Behrens, Peter (1994) Die Wirtschaftsverfassung der Europäischen Gemeinschaft. In: Gert Brüggemeier, Hrsg., Verfassungen für ein ziviles Europa. Baden-Baden: Nomos, 73-90.

Benz, Arthur/ Gerhard Lehmbruch (1996) Demokratische Legitimation regionaler Politik im europäischen Mehrebenensystem. Ms. Halle: Universität HalleWittenberg.

Bercusson, Brian et al. (1996) Soziales Europa - ein Manifest. Reinbek: Rowohlt.

Bickel, Alexander M. (1962) The Least Dangerous Branch. The Supreme Court at the Bar of Politics. Indianapolis: Bobbs-Merrill.

Böckenförde, Ernst Wolfgang (1997) Wenn der europäische Stier vom goldenen Kalb überholt wird. Die Politik in der ökonomischen Falle: Wirtschaftliche Einigung schafft noch keine politische Solidarität. In: Frankfurter Allgemeine Zeitung, 24.7.1997, 30.

Brennan, Geoffrey (1989) Politics with Romance: Towards a Theory of Democratic Socialism. In: Alan Hamlin/ Philip Pettit, Hrsg., The Good Polity. Normative Analysis of the State. Oxford: Basil Blackwell, 49-66.

Burley, Anne-Marie/ Walter Mattli (1993) Europe Before the Court. A Political Theory of Legal Integration. In: International Organization 47, 41-76.

Cerny, Philip G. (1990) The Changing Architecture of Politics. Structure, Agency, and the Future of the State. London: Sage. 
Cerny, Philip G. (1993) The Deregulation and Re-regulation of Financial Markets in a More Open World. In: Philip C. Cerny, Hrsg., Finance and World Politics.

Markets, Regimes and States in the Post-hegemonic Era. Aldershot: Elgar, 51-85.

Cohen, Joshua/ Joel Rogers (1992) Secondary Associations and Democratic Governance. In: Politics and Society 20, 393-472.

Cooke, Jacob E., Hrsg. (1961) The Federalist. Cleveland: Meridian Books.

Daele, Wolfgang van den/ Friedhelm Neidhardt (1996) "Regierung durch Diskussion" - Über Versuche, mit Argumenten Politik zu machen. In: Dieselben, Hrsg., Kommunikation und Entscheidung. Politische Funktionen öffentlicher Meinungsbildung und diskursiver Verfahren. WZB Jahrbuch 1966. Berlin: Sigma, 9-52.

Däubler, Wolfgang, Hrsg. (1989) Sozialstaat EG? Die andere Dimension des Binnenmarktes. Gütersloh: Bertelsmann Stiftung.

Delors, Jacques (1993) Entwicklungsperspektiven der Europäischen Gemeinschaft. In: Aus Politik und Zeitgeschichte B1/93, 3-9.

Dettling, Warnfried (1997) Die Erneuerung der Demokratie durch die (Bürger-) Gesellschaft. Vier Thesen zum Symposium "Wege zur Erneuerung der Demokratie" der Bertelsmann Stiftung. Berlin, 15. September 1997. Ms.: München.

Deutsch, Morton (1975) Equity, Equality, and Need: What Determines Which Value Will Be Used as the Basis of Distributive Justice? In: Journal of Social Issues 31, 137-149.

Dubiel, Helmut (1997) Der utopische Realismus der Demokratie. In: Merkur 51, 796804.

Ehmke, Horst (1961) Wirtschaft und Verfassung. Die Verfassungsrechtsprechung des Supreme Court zur Wirtschaftsregulierung. Karlsruhe: C.F. Müller.

Elias, Norbert (1987) Die Gesellschaft der Individuen. Frankfurt/M.: Suhrkamp.

Elster, Jon (1979) Ulysses and the Sirens. Studies in Rationality and Irrationality. Cambridge: Cambridge University Press.

Elster, Jon (1983) Sour Grapes. Studies in the Subversion of Rationality. Cambridge: Cambridge University Press.

Elster, Jon (1986) The Market and the Forum: Three Varieties of Political Theory. In: Elster, Jon / Aanund Hylland, Hrsg., Foundations of Social Choice Theory.

Cambridge: Cambridge University Press. 103-132.

Elster, Jon (1989) Nuts and Bolts for the Social Sciences. Cambridge: Cambridge University Press.

Elster, Jon (1989a) The Cement of Society. A Study of Social Order. Cambridge: Cambridge University Press.

Elster, Jon (1991) The Possibility of Rational Politics. In: David Held, Hrsg., Political Theory Today. Stanford: Stanford University Press, 115-142.

Friedrich, Carl J. (1937) Constitutional Government and Politics. New York: Harper. Gellner, Ernest (1983) Nations and Nationalism. Ithaca: Cornell University Press.

Graebner, William (1977) Federalism in the Progressive Era. A Structural Interpretation of Reform. In: Journal of American History 64, 331-357.

Grande, Edgar (1996) Demokratische Legitimation und europäische Integration. In: Leviathan 24, 339-360.

Grande, Edgar (1997) Abschied vom Nationalstaat? Entwicklungslinien moderner Staatlichkeit in Europa. Arbeitspapier Nr. 1/1997. Institut für Sozialwissenschaften. Technische Universität München.

Grimm, Dieter (1995) Braucht Europa eine Verfassung? München: Carl Friedrich von Siemens Stiftung.

Grofman, Bernard/ Scott L. Feld (1988) Rousseau's General Will: A Condorcetian Perspective. In: American Political Science Review 82, 567-576. 
Guéhenno, Jean-Marie (1994) Das Ende der Demokratie. München: Artemis \& Winkler.

Habermas, Jürgen (1973) Legitimationsprobleme im Spätkapitalismus. Frankfurt/M.: Suhrkamp.

Habermas, Jürgen (1992) Faktizität und Geltung. Beiträge zur Diskurstheorie des Rechts und des demokratischen Rechtsstaats. Frankfurt/M.: Suhrkamp.

Habermas, Jürgen (1995) Comment: "Does Europe Need a Constitution?" In: European Law Journal 1, 303-307.

Habermas, Jürgen (1996) Die Einbeziehung des Anderen. Studien zur politischen Theorie. Frankfurt/M.: Suhrkamp.

Heinelt, Hubert (1996) Die Transformation der Demokratie in der EU. Ms. Hannover: Universität Hannover.

Held, David (1993) Democracy: From City-states to a Cosmopolitan Order? In: David Held., Hrsg., Prospects for Democracy. North, South, East, West. Cambridge: Polity Press, 11-52.

Held, David (1995) Democracy and the New International Order. In: Daniele Archibugi/ David Held, Hrsg., Cosmopolitan Democracy. An Agenda for a New World Order. Cambridge: Polity Press, 96-120.

Hindess, Barry (1991) Imaginary Presuppositions of Democracy. In: Economy and Society 20, 173-195.

Hirst, Paul/ Grahame Thompson (1996) Globalization in Question. The International Economy and the Possibility of Governance. Cambridge: Polity Press.

Ipsen, Hans Peter (1972) Europäisches Gemeinschaftsrecht. Tübingen: Mohr. Joerges, Christian/ Jürgen Neyer (1997) From Intergovernmental Bargaining to Deliberative Political Processes: The Constitutionalization of Comitology. In: European Law Journal 3, 273-299.

Kaiser, Andre (1997) Types of Democracy: From Classical to New Institutionalism. In: Journal of Theoretical Politics 9, 419-444.

Kielmansegg, Peter Graf (1977) Volkssouveränität. Eine Untersuchung der Bedingungen demokratischer Legitimität. Stuttgart: Klett.

Kielmansegg, Peter Graf (1996) Integration und Demokratie. In: Markus Jachtenfuchs/ Beate Kohler-Koch, Hrsg., Europäische Integration. Opladen: Leske + Budrich, 47-72.

Kirchgässner, Gebhard (1992) Towards a Theory of Low-cost Decisions. In: European Journal of Political Economy 8, 305-320.

Ladeur, Karlheinz (1992) Die Autonomie der Bundesbank - ein Beispiel für die institutionelle Verarbeitung von Ungewißheitsbedingungen. In:

Staatswissenschaften und Staatspraxis 4, 486-508.

Larsson, Allan (1997) A Comment on the "Manifesto for Social Europe". In: European Law Journal 3, 304-307.

Leibfried, Stephan/ Paul Pierson, Hrsg. (1995) European Social Policy. Between Fragmentation and Integration. Washington, DC: Brookings.

Lindner, Clausjohann (1990) Kritik der Theorie der partizipatorischen Demokratie. Opladen: Westdeutscher Verlag.

Majone, Giandomenico (1996) Regulating Europe. London: Routledge.

Manin, Bernard (1987) On Legitimacy and Political Deliberation. In: Political Theory $15,338-368$.

Mayntz, Renate/ Fritz W. Scharpf (1995) Steuerung und Selbstorganisation in staatsnahen Sektoren. In: Dieselben, Hrsg., Gesellschaftliche Selbstregelung und politische Steuerung. Frankfurt/M.: Campus, 9-38.

Mestmäcker, Ernst-Joachim (1994) Zur Wirtschaftsverfassung der Europäischen Union. In: Rolf H. Hasse/ Josef Molsberger/ Christian Watrin, Hrsg., Ordnung in Freiheit. Festgabe für Hans Willgerodt zum 70. Geburtstag. Stuttgart: Klett, 263- 
292.

Mueller, Dennis C. (1996) Constitutional Democracy. Oxford: Oxford University Press.

Narr, Wolf-Dieter (1997) Das demokratische Fiasko der Europäischen Union. In: Carsten Schlüter-Knauer, Hrsg., Die Demokratie überdenken. Festschrift für Wilfried Röhrich. Berlin: Duncker \& Humblot, 251-260.

Neuman, Iver B. (1996) Self and Others in International Relations. In: European Journal of International Relations 2, 139-174.

Niedermayer, Oskar/ Richard Sinnot, Hrsg. (1995) Public Opinion and Internationalized Governance. Oxford: Oxford University Press.

Offe, Claus/ Ulrich K. Preuss (1991) Democratic Institutions and Moral Resources. In: David Held, Hrsg., Political Theory Today. Stanford: Stanford University Press, 143-171.

Ostrom, Elinor (1990) Governing the Commons. The Evolution of Institutions for Collective Action. Cambridge: Cambridge University Press.

Pierson, Paul (1994) Dismantling the Welfare State? Reagan, Thatcher, and the Politics of Retrenchment. Cambridge: Cambridge University Press.

Pitschas, Rainer (1994) Europäische Integration als Netzwerkkoordination komplexer Staatsaufgaben. In: Staatswissenschaften und Staatspraxis 5, 503-540.

Pollack, Mark A. (1997) Representing Diffuse Interests in EC Policymaking. In: Journal of European Public Policy, Special Issue. Im Erscheinen.

Riker, William H. (1982) Liberalism Against Populism. A Confrontation Between the Theory of Democracy and the Theory of Social Choice. San Francisco: W.H. Freeman.

Risse, Thomas (1997) Who Are We? A Europeanization of National Identities? Ms. Florenz: European University Institute.

Rousseau, Jean Jacques (1959) Der Gesellschaftsvertrag. Contrat Social. Stuttgart: Reclam.

Runciman, Walter G./ Amartya Sen (1965) Games, Justice, and the General Will. In: Mind 74, 554-562.

Sassoon, Donald (1996) Social Democracy at the Heart of Europe. London: Institute for Public Policy Research.

Scharpf, Fritz W. (1970) Demokratietheorie zwischen Utopie und Anpassung. Konstanz: Universitätsverlag.

Scharpf, Fritz W. (1985) Die Politikverflechtungs-Falle: Europäische Integration und deutscher Föderalismus im Vergleich. In: Politische Vierteljahresschrift 26, 323356.

Scharpf, Fritz W. (1992) Koordination durch Verhandlungssysteme: Analytische Konzepte und institutionelle Lösungen. In: Arthur Benz/ Fritz W. Scharpf/ Reinhard Zintl, Horizontale Politikverflechtung. Zur Theorie von Verhandlungssystemen. Frankfurt/M.: Campus, 51-96.

Scharpf, Fritz W. (1996) Negative and Positive Integration in the Political Economy of European Welfare States. In: Gary Marks et al., Governance in the European Union. London: Sage, 15-39.

Scharpf, Fritz W. (1997) Introduction: The Problem-Solving Capacity of Multi-Level Governance. In: Journal of European Public Policy 4, Special Issue, im Erscheinen.

Scharpf, Fritz W. (1997a) Globalisierung als Beschränkung der Handlungsmöglichkeiten nationalstaatlicher Politik. Discussion Paper 97/1. Köln: Max-Planck-Institut für Gesellschaftsforschung.

Scharpf, Fritz W. (1997b) Games Real Actors Play. Actor-Centered Institutionalism in Policy Research. Boulder: Westview.

Scharpf, Fritz W. (1997c) Balancing Positive and Negative Integration: The Regulatory Options for Europe. Working Paper. Http://www.mpifg.de. $<>$ 
Schmalz-Bruns, Rainer (1995) Reflexive Demokratie. Die demokratische Transformation moderner Politik. Baden-Baden: Nomos.

Schmidt, Susanne K. (1997) Behind the Council Agenda. The Supranational Shaping of Decisions. Discussion Paper 97/3. Köln: Max-Planck-Institut für Gesellschaftsforschung (im Erscheinen).

Schmitt, Carl (1963) Verfassungslehre. Berlin: de Gruyter (Nachdruck der Auflage von 1928).

Schwartz, Bernard (1957) The Supreme Court. Constitutional Revolution in Retrospect. New York: Ronald Press.

Smith, Anthony (1992) National Identity and the Idea of European Unity. In: International Affairs 68, 55-76.

Streeck, Wolfgang (1997) Industrial Citizenship Under Regime Competition: The Case of the "European Works Councils". In: Journal of European Public Policy 4, Special Issue, im Erscheinen.

Streit, Manfred E. (1996) Systemwettbewerb im europäischen Integrationsprozeß. In: Ulrich Immenga/ Wernhard Möschel/ Dieter Reuter, Hrsg., Festschrift für Ernst-

Joachim Mestmäcker zum siebzigsten Geburtstag. Baden-Baden: Nomos, 521-535.

Streit, Manfred E./ Werner Mussler (1994) The Economic Constitution of the European Community: From Rome to Maastricht. In: Constitutional Political Economy 5, 319-353.

Talmon, Jacob L. (1955) The Origins of Totalitarian Democracy. London: Secker \& Warburg.

Tsebelis, George (1995) Decision Making in Political Systems: Comparison of Presidentialism, Parliamentarism, Multicameralism, and Multipartism. In: British Journal of Political Science 25, 289-325.

Tsebelis, George/ Jeanette Money (1997) Bicameralism. Cambridge: Cambridge University Press.

Weber, Max (1956) Wirtschaft und Gesellschaft. Grundriß der verstehenden Soziologie. Vierte, neu herausgegebene Auflage, besorgt von Johannes Winckelmann. Tübingen: Mohr.

Weiler, J.H.H. (1995) Does Europe Need a Constitution? Reflections on Demos, Telos and the German Maastricht Decision. In: European Law Journal 1, 19-258.

Weiler, J.H.H. (1996) European Neo-constitutionalism: in Search of Foundations for the European Constitutional Order. In: Political Studies 44, 517-533.

Zürn, Michael (1996) Über den Staat und die Demokratie im europäischen Mehrebenensystem. In: Politische Vierteljahresschrift 37, 27-55.

\section{Endnoten}

1 Referat auf dem DVPW-Kongreß "Demokratie - eine Kultur des Westens", Bamberg, am 14.10.97.

2 Das historische Vorbild dafür boten die Vereinigten Staaten, wo die Einzelstaaten vor der "New-Deal-Revolution" von 1937 aus Angst vor der Standortkonkurrenz sich nicht in der Lage gesehen hatten, die Kinderarbeit zu verbieten und andere Regeln des Arbeitsschutzes durchzusetzen (Graebner 1977), während nach der Anerkennung einer Bundeskompetenz der Kongreß derartige Maßnahmen der Wirtschaftsregulierung bundeseinheitlich durchsetzen konnte (Ehmke 1961).

3 Es spricht auch nichts dagegen, daß positiv eingeschätzte Veränderungen in den Interaktionen zwischen Eltern und Kindern, zwischen Managern und Mitarbeitern, in Selbsthilfegruppen und in den transnational vernetzten Projekten von Greenpeace und Amnesty International als "radikale Demokratisierung" interpretiert werden (Dubiel 1997) - auch wenn nicht so recht klar ist, was damit gegen den "globalen Kapitalismus" ausgerichtet wäre.

4 So etwa Habermas in dem Aufsatz "Über den inneren Zusammenhang von 
Rechtsstaat und Demokratie" (Habermas 1996, 293-305).

5 Von einem radikal-individualistischen Standpunkt aus erschiene auch die erzwungene intertemporäre Selbstbindung problematisch (Elster 1979).

6 Zwar kann das Problem der zyklischen Instabilität durch institutionelle

Vorkehrungen ausgeschlossen werden, aber dadurch wird die Rationalität des so stabilisierten Ergebnisses nicht größer (Scharpf 1997b, Kap. 7).

7 Der gleichen Logik folgt das Maastricht-Urteil des Bundesverfassungsgerichts, wenn es (im Hinblick auf die nationale Demokratie) davon spricht, daß Staaten hinreichend bedeutsame eigene Aufgabenfelder verbleiben müssen, "auf denen sich das jeweilige Staatsvolk in einem von ihm legitimierten und gesteuerten Prozeß politischer Willensbildung entfalten und artikulieren kann, um so dem was es - relativ homogen - geistig, sozial und politisch verbindet, rechtlichen Ausdruck zu geben." 8 Auch für Rousseau, der gemeinhin der kollektivistischen Demokratietheorie zugeordnet wird, gibt es eine individualistische Lesart, sofern man nur voraussetzt, daß die beteiligten Individuen gleiche "objektive" Interessen haben, weil sie sich alle in der gleichen Lage (nämlich in einem symmetrischen Gefangenendilemma oder in der Konstellation eines "Versicherungsspiels") befinden (Runciman/Sen 1965).

9 Wenn es eine objektiv richtige Lösung gibt, und wenn die Wahrscheinlichkeit, daß die voneinander unabhängigen Einzelmeinungen das Richtige treffen, größer als Null ist, dann besagt das Condorcet-Jury-Theorem, daß mit der Zahl der dafür Votierenden auch die Chance steigt, daß das Abstimmungsergebnis das Richtige trifft (Grofman/ Feld 1988).

10 Nach dem Condorcet-Jury-Theorem würde das Abstimmungsergebnis sich dem Gemeinwohl ja schon dann annähern, wenn bei divergierenden Einzelinteressen und konvergierender Definition des Gemeinwohls die einzelnen Wähler mit geringer positiver Wahrscheinlichkeit für das Gemeinwohl votieren.

11 Bei solidarischer Interaktionsorientierung werden alle "Mixed-Motive-Spiele" zu reinen "Koordinationsspielen", bei denen die Beteiligten die gleichen Lösungen präferieren (Scharpf 1997b, ch. 4).

12 Der potentielle Bereich gemeinsamer Interessenverfolgung, der unter OutputKriterien selbst dann legitimiert werden kann, wenn auf seiten der Bürger ausschließlich egoistisch-rationale Orientierungen vorausgesetzt werden, ist also keineswegs so eng umschrieben, wie dies in der Habermas'schen Entgegensetzung einer wertorientierten deliberativen Politik und der bei interessegeleitetem Handeln allenfalls erreichbaren Kompromißlösungen erscheinen könnte (Habermas 1992, Kap. IV).

13 Kaiser (1997) stellt der majoritäten Demokratie die Verhandlungsdemokratie gegenüber, bei der er "consociational veto points", "delegatory veto points", "expert veto points" und "legitlatory veto points" unterscheidet. Diese nützliche

Klassifikation verdeckt allerdings den hier betonten Doppelaspekt der Ermöglichung und der Hemmung politischen Handelns.

14 Zur Bedeutung von "mechanisms" in den Sozialwissenschaften, vgl. Elster (1989, Kap.1; 1989a, viii) und Scharpf (1997b, Kap.2).

15 Die Nähe Elsters zur Habermas'schen Position ist offensichtlich, aber Elsters Argument ist empirisch gemeint und normativ weniger anspruchsvoll als der von Habermas postulierte Ausschluß von "nicht verallgemeinerungsfähigen Interessen" aus dem wahrheitsorientierten politischen Diskurs (Habermas 1973, 140-162).

16 Um allfällige Mißverständnisse auszuschließen, ist daran zu erinnern, da das Adjektiv "demokratisch" nicht für die Input-Legitimation reserviert werden darf.

17 Urteil vom 12.10.1993, 2 BvR 2134/92 und 2 BvR 2159/92, 37.

18 Der Mechanismus, der die Durchsetzung ermöglichte, wurde in dem bahnbrechenden Aufsatz von Anne-Marie Burley und Walter Mattli (1993) erklärt: In dem Maße, wie die Rechtsprechung des EuGH über das Vorlage-Verfahren von den 
nationalen Gerichten in die nationalen Rechtsordnungen integriert wird, müßten die nationalen Regierungen und parlamentarischen Mehrheiten den Rechtsstaat im eigenen Land in Frage stellen, um der richterlichen "Fortbildung" des Europarechts entgegenzutreten.

Copyright (C) 1997 Fritz W. Scharpf

No part of this publication may be reproduced or transmitted without permission in writing from the author.

Jegliche Vervielfältigung und Verbreitung, auch auszugsweise, bedarf der Zustimmung des Autors.

MPI für Gesellschaftsforschung, Paulstr. 3, 50676 Köln, Germany

MPIfG: MPIfG Working Paper 97/9

http://www.mpifg.de/pu/workpap/wp97-9/wp97-9.html

[Zuletzt geändert am 29.03.2007 10:59] 